\title{
Uso e integración de las TIC en asignaturas cuantitativas aplicadas: La experiencia de los estudios de informática y multimedia de la
}

\author{
Ángel A. Juan, $M^{a}$ Antonia Huertas, Cristina \\ Steegmann, Manuel Terradez \\ ajuanp@uoc.edu, mhuertassauoc.edu, \\ csteegmannauoc.edu, mterradezauoc.edu \\ Universitat Oberta de Catalunya (UOC)
}

\section{Introducción.}

Las tendencias actuales en la enseñanza identifican los avances tecnológicos como un valioso recurso capaz de acompañar el aprendizaje de distintas materias en cualquier etapa educativa. En este sentido, la aparición y difusión de Internet, junto con la evolución que ha experimentado el software estadístico y matemático en general han supuesto no sólo un incremento significativo en la capacidad productiva de estudiantes y profesores, favoreciendo la generación de nuevos y mejores recursos didácticos y de autoaprendizaje, sino también una nueva forma de crear y difundir conocimientos o experiencias cognitivas.

En nuestra opinión, existe aún un campo en el que el uso de los avances tecnológicos no se ha mostrado aún, al menos en este país, en todas sus posibilidades: la enseñanza de las matemáticas. Si bien es cierto que el uso de los ordenadores y de programas y lenguajes informáticos ha ido bastante ligado a la enseñanza de algunas materias tales como el cálculo numérico, la estadística y la investigación operativa, hasta la fecha aún se aprecia un cierto recelo a la hora de dar un paso más allá e introducir tales herramientas como componente básico en cursos de análisis matemático o de álgebra lineal, por sólo citar dos ejemplos, donde podrían ser realmente útiles. En este sentido, creemos necesario que estos recursos se integren en los currículos formativos como elementos importantes de los mismos.

Acorde con esta línea de pensamiento, compartida por profesores de diferentes universidades públicas y privadas, surgió el proyecto e-Math (www.uoc.edu/in3/e-math) del Internet Interdisciplinary Institute [W1]. La principal finalidad del e-Math es fomentar y difundir la utilización e integración de las herramientas tecnológicas actuales (Internet y software especializado) en los currículos de varias asignaturas cuantitativas aplicadas: Álgebra Lineal, Análisis Matemático, Estadística, Modelado y Simulación, Investigación Operativa, etc. Los resultados de este proyecto (material docente, artículos, conclusiones, etc.) son de difusión general vía la World Wide Web. Nuestra intención ha sido la de aprovechar las posibilidades que ofrece Internet para formar un cluster de conocimiento y poder así compartir nuestras experiencias y recursos docentes con otros grupos universitarios afines. Por ello, en el fondo el e-Math es un proyecto de gestión del conocimiento destinado a potenciar la calidad docente de las asignaturas cuantitativas aplicadas.

Nuestra experiencia docente en la UOC nos lleva a concluir que la integración adecuada de las Tecnologías de la Información y la Comunicación (TIC) en el diseño curricular de las asignaturas cuantitativas aplicadas favorece una mayor dosis de participación, creatividad, aprendizaje significativo y motivación por parte del estudiante. Esto permite establecer, conceptualmente hablando, objetivos más ambiciosos y afrontar el análisis de problemas más realistas y complejos que, 
de otra forma, resultarían muy difíciles -sino imposibles- de trabajar en un curso universitario de carácter introductorio, bien por su dificultad intrínseca, bien por la falta de tiempo suficiente. Por otro lado, el uso de software científico permite, también, liberar al estudiante de cierta inversión temporal en aprender técnicas de cálculo repetitivas, lo que le puede permitir dedicarse más a trabajar y comprender los conceptos, las ideas y las aplicaciones. Las ventajas del uso de las TIC son entonces enormes e innegables.

No hay que olvidar, sin embargo, que como toda tecnología, también el uso irracional de Internet y de software científico puede tener aspectos contraproducentes, sobre todo en las carreras en las que un conocimiento matemático conceptualmente débil puede tener consecuencias poco favorables. Por ello, siempre es importante que el equipo de profesores actúe como guía del aprendizaje, definiendo un punto de equilibrio - probablemente dinámico- entre el empleo combinado de las TIC y de los métodos tradicionales, e incentivando al estudiante para que éste realice siempre el esfuerzo de analizar la coherencia de los resultados que está obteniendo y de comprender los fundamentos teóricos y conceptuales en los que se basan dichos resultados.

\section{Experiencia de los estudios de Informática y Multimedia de la UOC.}

Los estudiantes de las titulaciones de Informática y multimedia de la UOC utilizan Internet y varios programas informáticos en las asignaturas del ámbito matemático o asignaturas cuantitativas. Con el propósito de fomentar este uso y darle el máximo valor añadido posible, los profesores de la UOC han ido elaborando una serie de materiales didácticos en los que las TIC juegan un papel relevante. Dichos materiales, que van siendo introducidos por los profesores en las respectivas aulas del Campus Virtual y/o en la página web del e-Math, complementan perfectamente al material principal de cada asignatura (módulos didácticos, generalmente de carácter más teórico que aplicado) y están compuestos por las Guías de Estudio, los Math-blocks, y las Pruebas de Evaluación Continua (que tienen una doble función formativa-evaluativa). De forma más flexible, los profesores de cada asignatura también proporcionan a los estudiantes ejercicios clave, plantillas interactivas prediseñadas y prácticas a resolver utilizando recursos disponibles en Internet o con la ayuda de algún software especializado. A continuación se describen con más detalle algunos de los recursos docentes citados anteriormente:

Guías de estudio (GES): Aunque su estructura es variable en función de la tipología de la asignatura, suelen contener un listado de objetivos y un resumen de los conceptos más importantes del tema que se trata. En la mayoría de los casos, el grueso de las GES suele estar constituido por ejercicios tipo resueltos en detalle y de forma dual, tanto usando métodos tradicionales como recurriendo a la ayuda de programas informáticos. Finalmente, suelen incluir también una lista de ejercicios propuestos. Las GES, que constituyen uno de los recursos más valorados por los propios estudiantes de la UOC, refuerzan el material didáctico de la asignatura y orientan el proceso de aprendizaje de la misma.

Math-blocks: Son unidades didácticas accesibles desde la página del proyecto e-Math. Suelen estar en formato PDF (para maximizar su portabilidad y la posibilidad de impresión), son altamente autocontenidas, y en ellas se potencia el uso de Internet (vía la selección de recursos de la web) y de software especializado (mediante el estudio de casos y ejemplos resueltos). En muchos casos, suelen también ir acompañadas de ficheros asociados de carácter interactivo o complementario (ficheros xls, avi, mcd, mtw, etc.)

Pruebas de evaluación continua (PECs): La evaluación continua (EC) se lleva a cabo mediante la realización de pruebas virtuales que los estudiantes resuelven en casa y envían a su profesor dentro de un plazo preestablecido en el Plan Docente (sylabus) de la asignatura. La estructura específica de cada PEC depende de la asignatura y del tema en cuestión, si bien se trata, en la medida de lo posible, de estimular la creatividad de los estudiantes, las consultas a recursos web previamente seleccionados por los profesores, la aplicación de los conceptos y el tratamiento dual de los ejercicios propuestos, combinando y relacionando la resolución tradicional con la resolución auxiliada por el uso de algún software especializado. 
Al final del semestre los estudiantes deben realizar también un examen final o una prueba presencial que valide la autoría de las actividades realizadas a lo largo de la EC. La estructura de dicha prueba final depende de la asignatura en cuestión, pero en la mayoría de los casos consta de una parte teórica -basada en conceptos, ideas y métodos clave de la asignatura-, y otra práctica -en la que dichos conceptos, ideas y métodos son aplicados sobre datos reales con ayuda del software utilizado durante el semestre-.

\subsection{Uso de las TIC en Matemática Discreta}

El contenido de la asignatura Matemática Discreta se divide en dos partes: en la primera se estudian técnicas básicas de cálculo combinatorio y en la segunda los fundamentos básicos de la teoría de grafos.

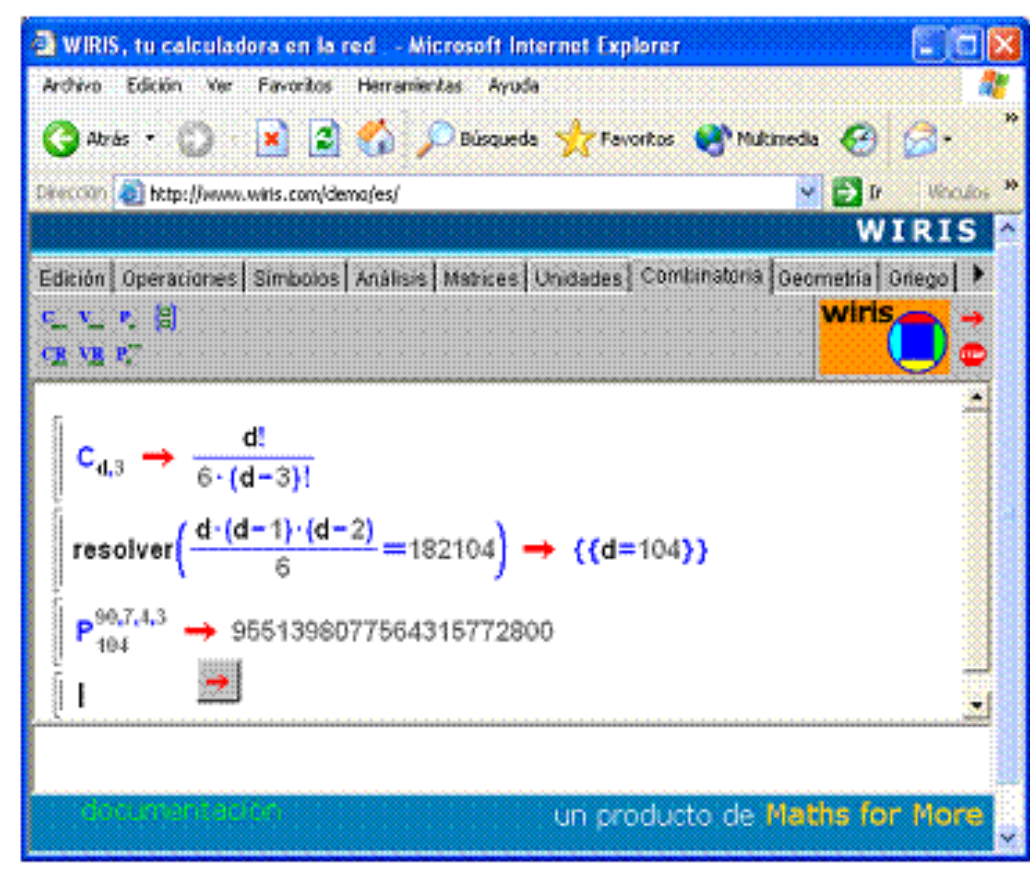

Fig. 1: cakulacora on-line Wirs

Tanto en la resolución de los ejercicios y problemas propuestos en el material de la asignatura como en las prácticas desarrolladas en el proceso de evaluación continua, los estudiantes cuentan con herramientas tecnológicas como el software especializado Mathcad o la calculadora simbólica online MatWiris [W2]. Estos recursos les facilita la realización de cálculos complejos y repetitivos en algunos casos- y les permite verificar $y$ contrastar los resultados obtenidos.

En la segunda parte, teoría de grafos, se utiliza además el software especializado Applet JavaGraph que permite a los estudiantes practicar los diferentes algoritmos de exploración de grafos que han estudiado de forma teórica. JavaGraph es una aplicación en Java creada por profesores de los Estudios de Informática de la UOC, de forma que se adapta y se integra completamente en el diseño de la asignatura. El applet, junto con una breve descripción de los diferentes algoritmos y actividades guiadas para su aprendizaje, forma parte de un material web que los estudiantes tienen en el aula virtual.

JavaGraph refuerza la práctica en la aplicación de los algoritmos ya que el estudiante puede disponer, de forma inmediata, de la corrección de sus ejercicios. También le ayuda a profundizar y consolidar lo que ha estudiado de forma teórica, realizando sus propias pruebas y descubrimientos (simulaciones interactivas).

Además, en esta asignatura los estudiantes utilizan diferentes enlaces de Internet que, de una u otra forma, les facilitan la asimilación de los contenidos y hacen que el proceso de aprendizaje sea más dinámico y creativo. Entre dichos enlaces se encuentran libros on-line y programas especializados, así como material docente elaborado por los propios profesores de la UOC.

\subsection{Uso de las TIC en Análisis y Álgebra.}

En estas asignaturas contamos con software como Mathcad, Scientific Notebook (SN) y la calculadora simbólica on-line MatWiris. Estos programas 


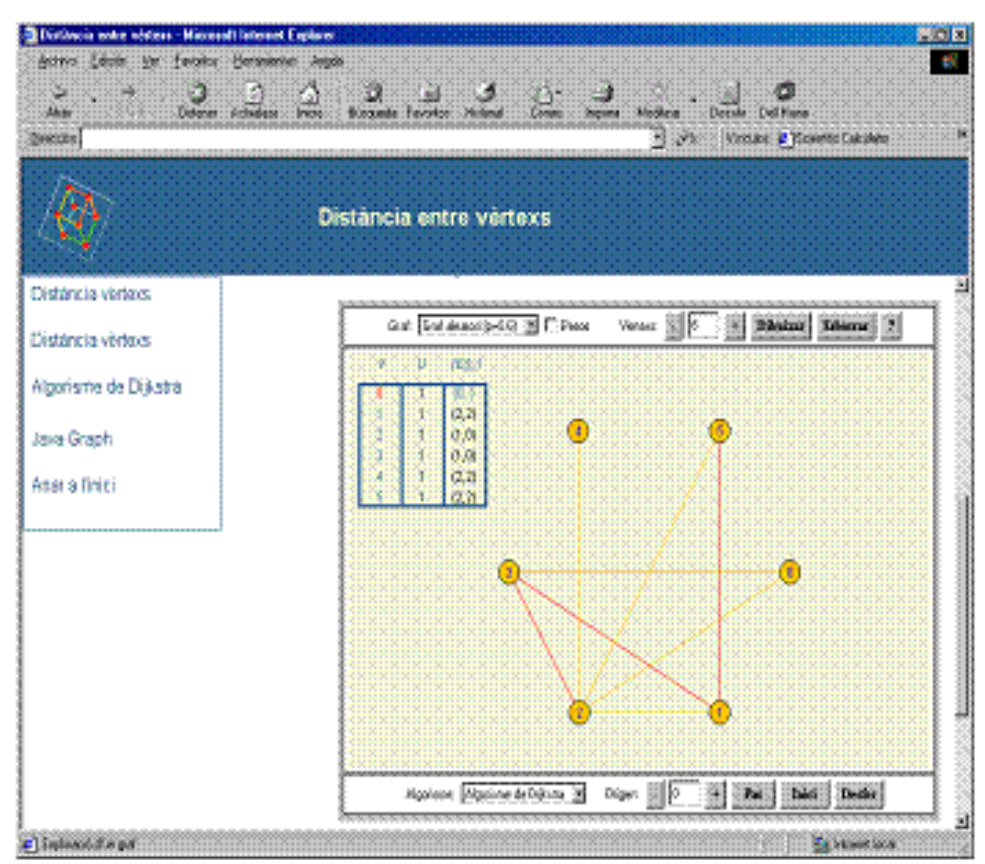

Fig. 2: JavaGraph
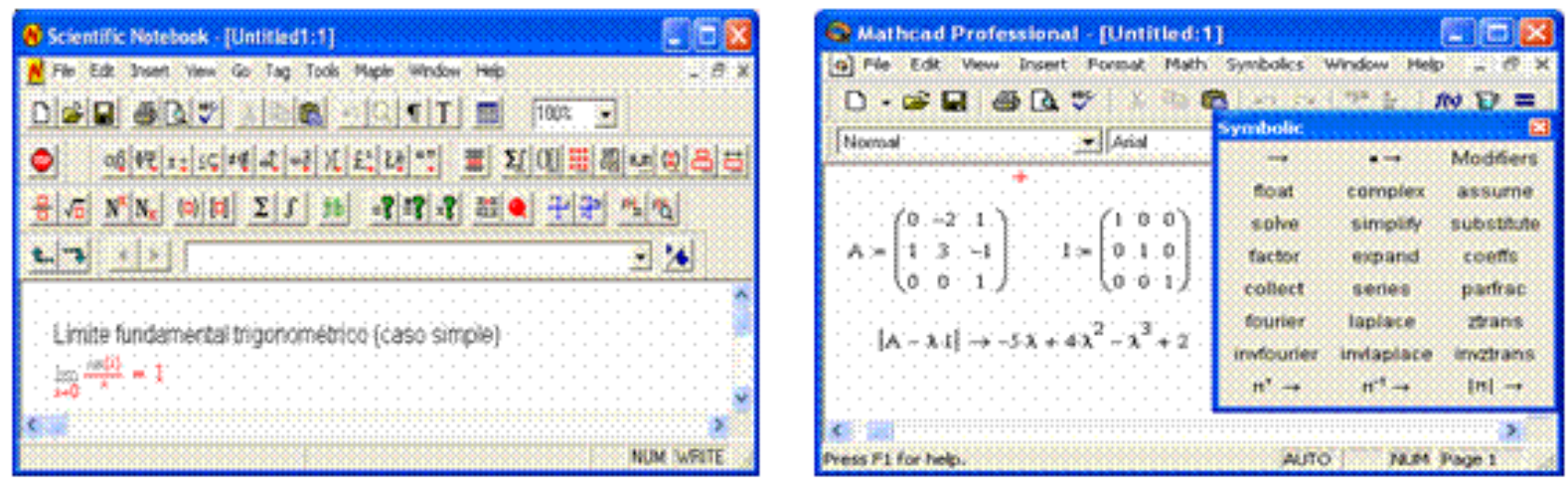

Fig. 3: Scientific Notebook y Mathcad nos sirven como editores de ecuaciones (y texto) y como herramientas de cálculo numérico y simbólico.

Con el uso de programas como Mathcad o SN el estudiante dispone, en un mismo entorno, de una potente herramienta que le permite editar ecuaciones, textos e imágenes $\mathrm{y}$, al mismo tiempo, realizar tanto cálculos numéricos y simbólicos como representaciones gráficas en 2D y 3D.

A modo de ejemplo, tareas simbólicas como el cálculo de determinantes con parámetros y su posterior simplificación, el cálculo de derivadas parciales, el polinomio de Taylor o el cálculo del límite de su resultado, se pueden efectuar fácilmente con estos programas.
En aspecto numérico permiten, por ejemplo, calcular extremos absolutos de una función de varias

variables, raíces de polinomios, resolver sistemas de ecuaciones o los valores y vectores propios de una matriz diagonalizable.

La capacidad de edición y representación en 2D y 3D, la facilidad con que se pueden diseñar y activar animaciones, así como las posibilidades de programación incorporadas hacen de este tipo de software herramientas didácticas muy completas y aptas para todos los niveles universitarios.

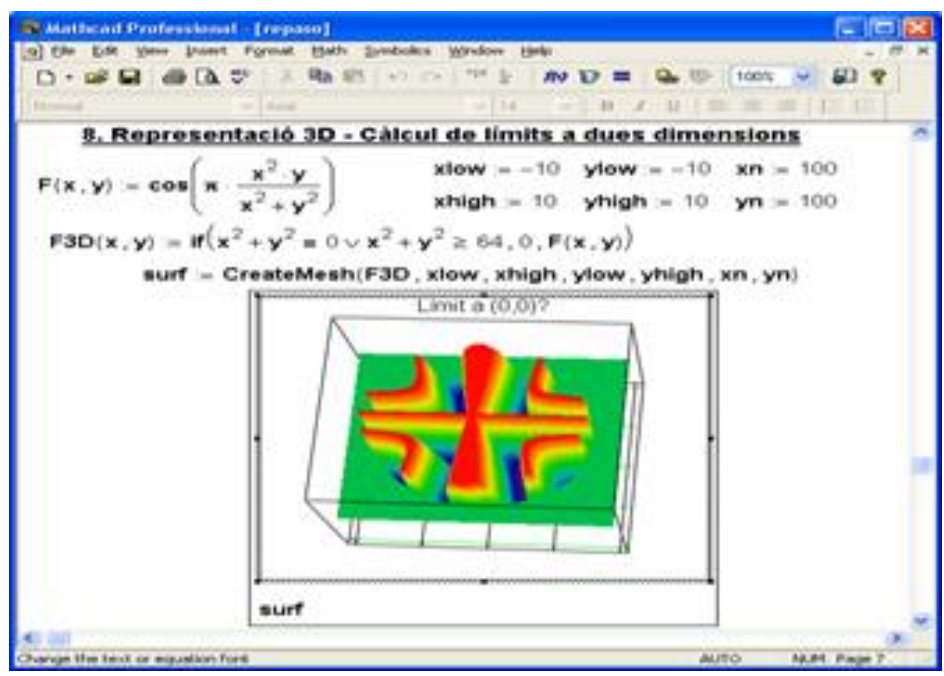




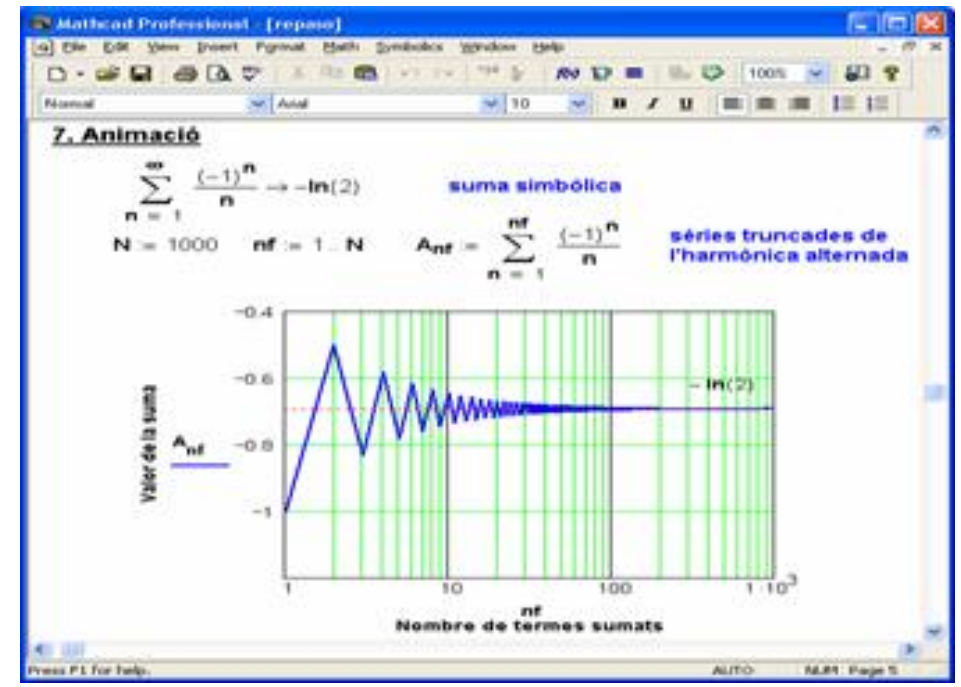

En la imagen se muestra un ejemplo de representación en 3D realizada con Mathcad. Una vez definida la función, basta con crear una red de puntos que son representados posteriormente. La posibilidad de rotar la imagen en los tres grados de libertad angulares permite determinar visualmente límites, máximos y mínimos, etc.

En cuanto a aspectos didácticos se refiere, las animaciones, totalmente exportables a otros entornos, son de una importancia relevante, puesto que permiten ilustrar y aclarar conceptos tales como la convergencia de una serie -que, debido al elevado número de operaciones, no se puede ilustrar intuitivamente sin ayuda de un software-. En la imagen se muestran sumas parciales de la serie armónica alternada.

Los estudiantes disponen de manuales de uso de los programas, con ejemplos prácticos detallados y ejercicios guiados que les ayudan a familiarizarse rápidamente con el uso del editor matemático y con las herramientas de cálculo. Este material se integra perfectamente en las asignaturas, ya que está estructurado según el índice y programa de éstas.
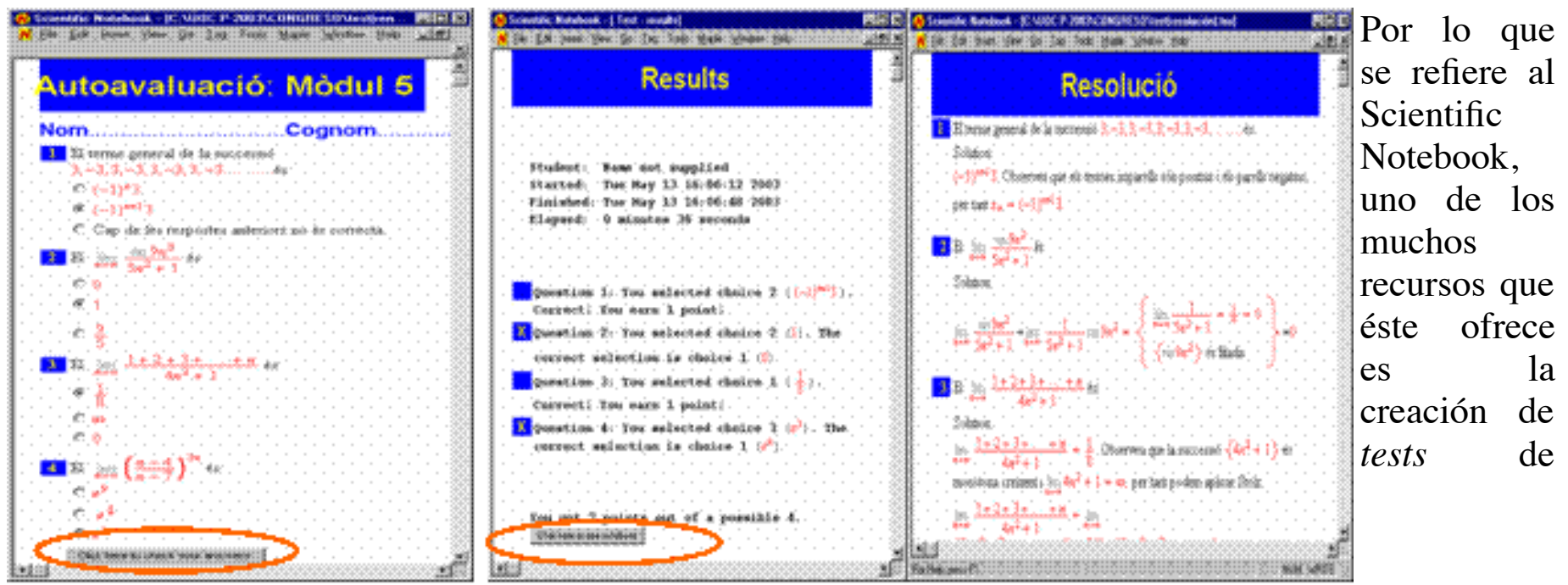

Fig. 5: ejempios de uso del Scientific Notebook

autoevaluación. Tanto en Álgebra como en Análisis se han diseñado este tipo de tests de manera que aparezca una prueba distinta cada vez que se abre el fichero-plantilla. Ello permite al estudiante comprobar su nivel de aprendizaje tantas veces como quiera. Cada test consta de tres páginas: en la primera, el alumno marca la respuesta que cree correcta a cada una de las preguntas planteadas, en la segunda comprueba si estas respuestas han sido o no correctas y la puntuación obtenida y en la tercera puede consultar la resolución completa de cada uno de los ejercicios propuestos en el test. Este recurso metodológico, elaborado con el $\mathrm{SN}$, constituye una fuente casi inagotable de material de 
autoaprendizaje y autoevaluación.

\subsection{Uso de las TIC en estadística.}

Los estudiantes de las Ingenierías de Informática de la UOC utilizan el software Minitab en la asignatura de estadística. Para tal fin, el equipo de profesores de la UOC ha ido desarrollando toda una serie de materiales didácticos en los que el uso del programa juega un papel relevante. Dichos materiales, disponibles en la web del Proyecto e-Math, están compuestos por un conjunto de Mathblocks que tratan de potenciar la realización de prácticas con este software así como la utilización de recursos de Internet en el aprendizaje de la estadística (applets de Java, artículos de aplicaciones diversas, etc.). La finalidad de todo este esfuerzo docente no es otra que la de facilitar el aprendizaje de conceptos y métodos estadísticos aplicados, sin por ello perder el rigor que toda asignatura cuantitativa debe tener: el uso correcto de cualquier software estadístico requiere que el investigador posea una base teórica adecuada, de forma que ésta le capacite para analizar con profundidad los resultados y extraer las conclusiones correctas.

\subsubsection{Características destacables del Minitab}

Este paquete estadístico posee ciertas propiedades técnicas que lo hacen ser especialmente útil en el aprendizaje de la estadística aplicada. Entre ellas, cabría destacar un entorno agradable y de fácil uso, su integración en un único paquete -que incluye todos los aspectos necesarios para la formación universitaria: análisis descriptivo, contrastes de hipótesis, regresión lineal y no lineal, series temporales, análisis de tiempos de fallo, control de calidad, análisis factorial, ANOVA, análisis cluster, etc.-, una excelente capacidad gráfica, total compatibilidad con las hojas de cálculo, herramientas de gestión de proyectos, conectividad ODBC para bases de datos, y un potente lenguaje de macros que permite automatizar y personalizar muchas de las tareas. A estas características técnicas, hay que añadir la enorme cantidad de bibliografía existente -más de 400 libros- en la que se explican conceptos y métodos estadísticos y sus aplicaciones a la ingeniería y a las ciencias económicas, sociales, de la información y la comunicación, etc.

\subsubsection{Ventajas de usar Minitab en la formación universitaria}

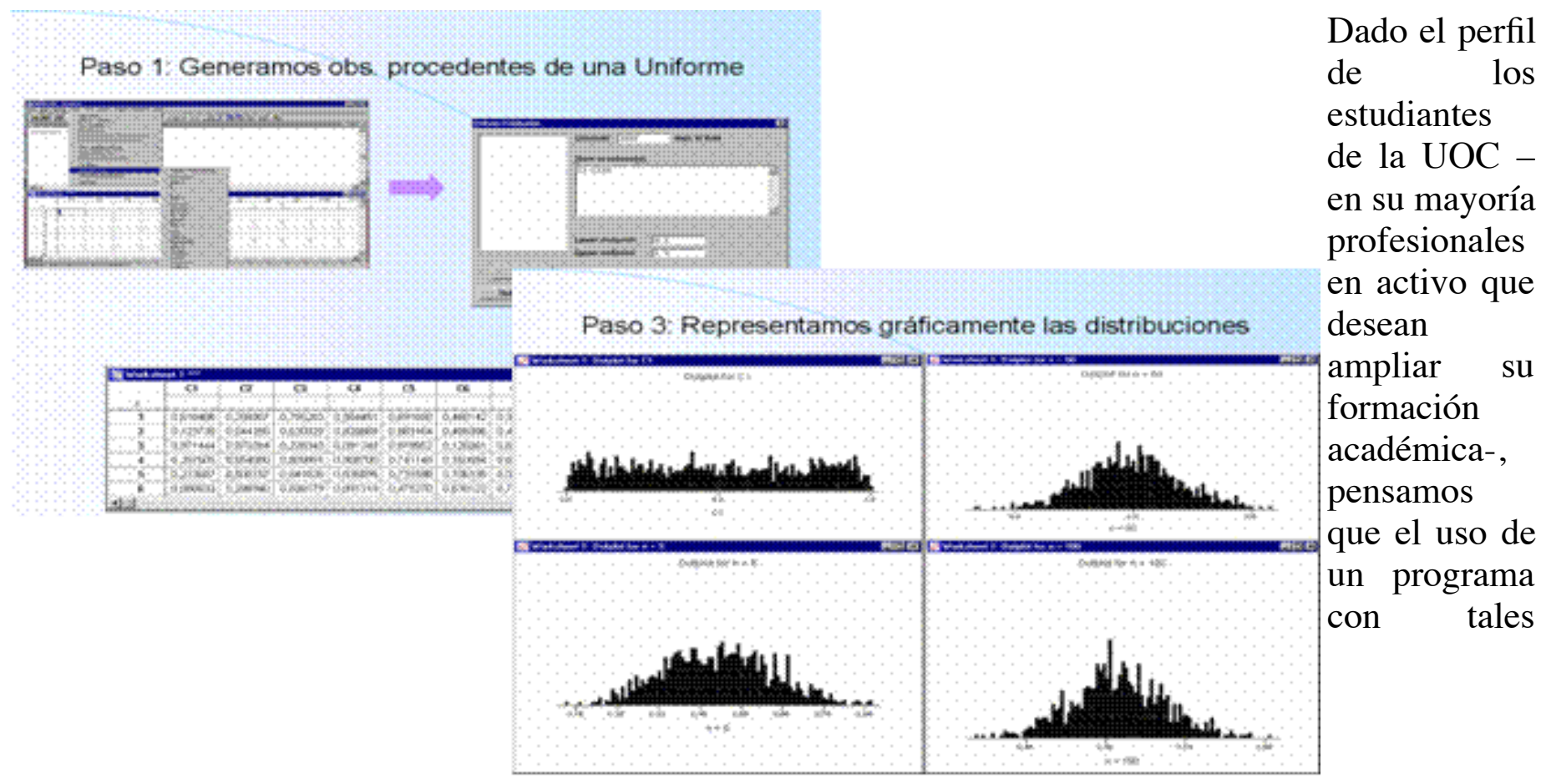

Fig. 6: ejempios de uso del Minitab

características aporta un gran valor añadido a aquellos que cursan asignaturas de estadística aplicada, ya que les permite adquirir destreza en el manejo de un programa profesional de idénticas o similares 
características al que se encontrarán en su lugar de trabajo. Por otro lado, no cabe duda de que las necesidades estadísticas de cualquier empresa requieren del uso de herramientas informáticas como Minitab. Esta mayor conectividad entre la universidad y las necesidades del mundo laboral tiende a incrementar el interés y la motivación de los estudiantes por la estadística, la cual se convierte en una ciencia de considerable aplicabilidad en sus entornos cotidianos de trabajo. Pero, además, hay otra razón de peso por la cual el uso de un paquete estadístico como Minitab puede ser altamente recomendable en cualquier curso de estadística aplicada, en especial cuando se trate de estudios a distancia o virtuales en los que se carece del contacto físico con los profesores: con un PC y Minitab, el estudiante dispone de un potente

laboratorio personal con el cual poder experimentar -preferentemente de forma guiada por su profesor- con los conceptos, métodos y aplicaciones de la estadística. En este sentido, una de las prácticas que solemos pedir a nuestros estudiantes es la verificación experimental de un resultado tan importante como es el Teorema del Límite Central, para lo cual el estudiante deberá generar -con ayuda del programa- diferentes conjuntos de observaciones aleatorias y comprobar, a partir de éstas, el comportamiento de la media muestral conforme aumenta el tamaño de la muestra.

A lo largo del semestre se proponen varias prácticas, en las que los conceptos y métodos estadísticos son aplicados sobre datos reales con ayuda de Minitab. Habitualmente, dichas prácticas consisten en la obtención de determinados datos vía internet -bases de datos del INE, BCE, OJD, etc.-, y el tratamiento de los mismos con el fin de obtener unos resultados. Posteriormente, cada estudiante debe analizar e interpretar dichos resultados, respondiendo a una serie de preguntas convenientemente formuladas y estructuradas por el equipo de profesores.

\section{Lineas de trabajo futuro}

En su fase actual, el proyecto e-Math cuenta ya con una excelente colección de documentos asociados a diferentes asignaturas cuantitativas de carácter aplicado. Uno de nuestros principales desafíos de futuro es seguir enriqueciendo los fondos documentales mediante la actualización de documentos ya existentes y, sobre todo, a través de la incorporación de nuevos materiales docentes -fruto de nuestra experiencia académica en la UOC.

En lo referente a las asignaturas que impartimos, nos proponemos ir perfeccionando los materiales didácticos con que contamos como resultado de la práctica docente y del intercambio de experiencias con otros grupos universitarios. Una de las consecuencias del uso de las TIC y de la evolución de los materiales didácticos será, posiblemente, el cambio en la naturaleza misma de las matemáticas que se enseñan y, sobre todo, en cómo y para qué se enseñan.

\section{Conclusiones}

A partir de nuestra experiencia, pensamos que el uso de los recursos disponibles en Internet y la utilización de un software adecuado contribuyen en gran medida a que la enseñanza de las matemáticas sea más asequible, práctica y creativa.

Entre las ventajas que supone la metodología propuesta en el artículo destacamos las siguientes: nos permite plantear objetivos didácticos más ambiciosos; abordar problemas prácticos que de otro modo serían inabordables; utilizar nuevos y mejores recursos didácticos; establecer una mayor conectividad entre la enseñanza universitaria y las necesidades del mundo laboral y lograr una mayor motivación de los estudiantes por el estudio de las matemáticas y de la estadística.

\section{Referencias}

\section{Artículos}

ALEMÁN DE SÁNCHEZ, A. (1999): La enseñanza de la matemática asistida por computadora. 
www.utp.ac.pa/articulos/ensenarmatematica.html

ÁLVAREZ, M.; et al (2002): Matemáticas, software y e-learning: La experiencia de la UOC con Mathcad. Fórum Tecnológico, número 3. ISSN: 1579-3818.

BERGER, T.; POLLATSEK, H. (2001): Mathematics and Mathematical Sciences in 2010: What Should Students Know?. www.maa.org/news/students2010.html

JUAN, A.; BAUTISTA, G. (2001): Didáctica de las matemáticas en enseñanza superior: la utilización de software especializado. www.uoc.edu/web/esp/art/uoc/0107030/mates.html

LANGTANGEN, H.; TVEITO, A. (2000). How Should We Prepare the Students of Science and Technology for a Life in the Computer Age?. Mathematics Unlimited - 2001 and Beyond. Springer Verlag.

SCHWARTZ, J. L. (1999). Can Technology Help Us Make the Mathematics Curriculo Intellectually Stimulating and Socially Responsible? International Journal of Computers for Mathematical Learning. Vol. 4, Núm. 2/3, p. 99-119.

SUTHERLAND, R. (1999). Didactical Complexity of Computational Environments for the Learning of Mathematics. International Journal of Computers for Mathematical Learning. Vol. 4, núm. 1, p. 126.

VILA, A.; et al (2002): ¿Cómo aprender estadística en un entorno virtual?: La experiencia de la UOC con Minitab. Fórum Tecnológico, número 2. ISSN: 1579-3818.

WILSON, J.W. (1998): Technology in Mathematics Teaching and Learning. http://jwilson.coe.uga.edu/Texts.Folder/Tech/Technology.Paper.html

\section{Enlaces web}

[W1] Internet Interdisciplinary Institute www.uoc.edu/in3

[W2] Calculadora on-line MatWiris www.wiris.com

[W3] Journal For Research In Mathematics Education (JRME)

Www.nctm.org/jrme/issues/2001/05/

[W4] International Journal of Computers for Mathematical Learning www.wkap.nl/kapis/CGI-BIN/WORLD/journalhome.htm?1382-3892

[W5] Journal of Computers in Mathematics and Science Teaching (JCMST) www.aace.org/pubs/jcmst/

[W6] Statistics on the web www.execpc.com/ helberg/statistics.html

(C) Ediciones Universidad de Salamanca 